\title{
Passivity-based Visual Force Feedback Control for Eye-to-Hand Systems
}

\author{
Hiroyuki Kawai ${ }^{1}$, Toshiyuki Murao ${ }^{2}$ and Masayuki Fujita ${ }^{3}$ \\ ${ }^{1}$ Kanazawa Institute of Technology, Ishikawa \\ Japan \\ ${ }^{2}$ Advanced Institute of Industrial Technology, Tokyo \\ Japan \\ ${ }^{3}$ Tokyo Institute of Technology, Tokyo \\ Japan
}

\section{Introduction}

Robotics and intelligent machines need sensory information to behave autonomously in dynamical environments. Visual information is particularly suited to recognize unknown surroundings. Vision based control of robotic systems involves the fusion of robot kinematics, dynamics, and computer vision to control the motion of the robot in an efficient manner. The combination of mechanical control with visual information, so-called visual feedback control or visual servoing, is important when we consider a mechanical system working under dynamical environments (Chaumette \& Hutchinson, 2008).

For the theoretically problem of three dimensional(3D) visual servo control based on the robot control theory, (Kelly et al., 2000) considered a simple image-based controller under the assumption that the objects' depths are known. (Chen et al., 2007) addressed the field-ofview problem for 3D dynamic visual feedback system using an image-space navigation function. In our previous works, we discussed the dynamic visual feedback control for 3D target tracking based on passivity (Fujita et al., 2007) (Murao et al., 2008). On the other hand, applications of visual feedback system are also increasing in many fields. For example, recent applications of visual feedback system include the autonomous injection of biological cells (Yu \& Nelson, 2001), laparoscopic surgery (Omote et al., 1999) and others. Although visual information is necessary in order to recognize environments, only visual information is not enough to complete tasks in these applications. For example, not only visual information but also force information are needed to inject DNA to biological cells. Hence, integrating visual feedback control with force control is important for the modern robot.

(Xiao et al., 2000) developed sensor fusion scheme for controlling an end-effector to follow an unknown trajectory on a contact surface. (Baeten et al., 2003) addressed a hybrid control structure for the eye-in-hand vision and force control. Although many practical methods are reported with experimental results, rigorous results have hardly been obtained in terms of the nonlinear control aspects. For this problem, (Dean-Leon et al., 2006) has combined image-based visual feedback control with force control and discussed the stability of the 
nonlinear system. The authors have proposed passivity based visual force feedback control law for force control with target tracking (Kawai et al., 2007). Although these control laws guarantee Lyapunov stability and are effective for the visual force feedback system, they are restricted to planar manipulators.

This chapter deals with $3 \mathrm{D}$ visual force feedback control for eye-to-hand systems as depicted in Fig. 1. In our proposed method, we can control not only the position but also the orientation of the robot hand with a contact force in the visual force feedback system. The main contribution of this chapter is to show that the 3D visual force feedback system has the passivity which allows us to prove stability in the sense of Lyapunov. Both the passivity of the manipulator dynamics and the passivity of the visual feedback system are preserved in the $3 \mathrm{D}$ visual force feedback system. Finally simulation results are shown to verify the stability of the proposed method.

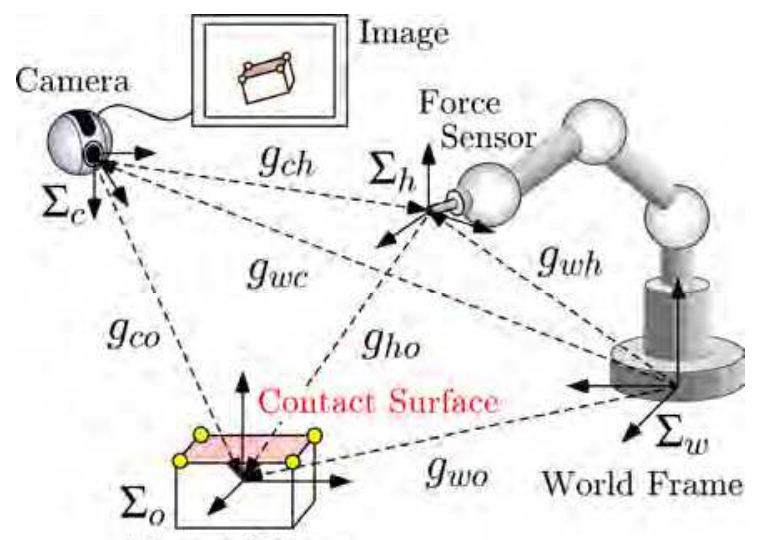

Target Object

Fig. 1. Visual force feedback system with an eye-to-hand configuration

\section{Visual Feedback System}

This section mainly reviews our previous works (Fujita et al., 2007) (Murao et al., 2008) via the passivity based visual feedback control. Throughout this chapter, we use the notation $e^{\hat{\xi} \theta_{a b} \in \mathfrak{R}^{3 \times 3}}$ to represent the change of the principle axes of a frame $\Sigma_{b}$ relative to a frame $\Sigma_{a} \cdot \xi_{a b} \in \mathfrak{R}^{3}$ specifies the direction of rotation and $\theta_{a b} \in \mathfrak{R}$ is the angle of rotation. For simplicity we use $\hat{\xi} \theta_{a b}$ to denote $\hat{\xi}_{a b} \theta_{a b}$. The notation ' $\wedge$ ' (wedge) is the skew-symmetric operator such that $\hat{\xi} \theta=\xi \times \theta$ for the vector cross-product $\times$ and any vector $\theta \in \mathfrak{R}^{3}$. The notation ' $\vee$ ' (vee) denotes the inverse operator to ' $\wedge$ ', i.e., so(3) $\rightarrow \mathfrak{R}^{3}$. Recall that a skewsymmetric matrix corresponds to an axis of rotation (via the mapping $a \mapsto \hat{a}$ ). We use the $4 \times 4$ matrix

$$
g_{a b}=\left[\begin{array}{cc}
e^{\hat{\xi} \theta_{a b}} & p_{a b} \\
0 & 1
\end{array}\right]
$$


as the homogeneous representation of $g_{a b}=\left(p_{a b}, e^{\hat{\xi} \theta_{a b}}\right) \in S E(3)$ describing the configuration of a frame $\Sigma_{b}$ relative to a frame $\Sigma_{a}$. The adjoint transformation associated with $g_{a b}$ is denoted by $\operatorname{Ad}_{\left(g_{a b}\right)}$ (Murray et al., 1994).

\subsection{Basic Representation for Visual Feedback System}

Visual feedback systems with an eye-to-hand configuration typically use four coordinate frames which consist of a world frame $\Sigma_{w}$, a target object frame $\Sigma_{o}$, a camera frame $\Sigma_{c}$ and a hand (end-effector) frame $\Sigma_{h}$ as in Fig. 1. Then, $g_{w h}=\left(p_{w h}, e^{\hat{\xi} \theta_{w h}}\right) \in S E(3)$, $g_{w c}=\left(p_{w c}, e^{\hat{\xi} \theta_{w c}}\right) \in S E(3)$ and $g_{w o}=\left(p_{w o}, e^{\hat{\xi} \theta_{w o}}\right) \in S E(3)$ denote the rigid body motion from $\Sigma_{w}$ to $\Sigma_{h}$, from $\Sigma_{w}$ to $\Sigma_{c}$ and from $\Sigma_{w}$ to $\Sigma_{o}$, respectively. Similarly, the relative rigid body motion from $\Sigma_{c}$ to $\Sigma_{h}$, from $\Sigma_{c}$ to $\Sigma_{o}$ and from $\Sigma_{h}$ to $\Sigma_{o}$ can be represented by $g_{c h}=\left(p_{c h}, e^{\hat{\xi} \theta_{c h}}\right) \in S E(3), g_{c o}=\left(p_{c o}, e^{\hat{\xi} \theta_{c o}}\right) \in S E(3)$ and $g_{h o}=\left(p_{h o}, e^{\hat{\xi} \theta_{h o}}\right) \in S E(3)$, respectively, as shown in Fig. 1. The objective of the visual feedback control is to bring the actual relative rigid body motion $g_{h o}$ to a given reference $g_{d}=\left(p_{d}, e^{\hat{\xi} \theta_{d}}\right)$ which is constant in this chapter. In other words, our goal is to determine the motion of hand by using the visual information. The relative rigid body motion from $\Sigma_{c}$ to $\Sigma_{o}$ can be led by using the composition rule for rigid body transformations ((Murray et al., 1994), Chap. 2, pp. 37, eq. (2.24)) as follows:

$$
g_{c o}=g_{w c}^{-1} g_{w o}
$$

The relative rigid body motion involves the velocity of each rigid body. To this aid, let us consider the velocity of a rigid body as described in (Murray et al., 1994). We define the body velocity of the camera relative to the world frame $\Sigma_{w}$ as $V_{w c}^{b}=\left[v_{w c}^{T} \omega_{w c}^{T}\right]^{T}$, where $v_{w c}$ and $\omega_{w c}$ represent the velocity of the origin and the angular velocity from $\Sigma_{w}$ to $\Sigma_{c}$, respectively ((Murray et al., 1994), Chap. 2, eq. (2.55)).

Differentiating (2) with respect to time, the body velocity of the relative rigid body motion $g_{c o}$ can be written as follows (See (Fujita et al., 2007)):

$$
V_{c o}^{b}=-\mathrm{Ad}_{\left(g_{c o}^{-1}\right)} V_{w c}^{b}+V_{w o}^{b}
$$

where $V_{w o}^{b}$ is the body velocity of the target object relative to $\Sigma_{w}$. In the case of the eye-tohand configuration, i.e. $V_{w c}^{b}=0$, the model of the relative rigid body motion $g_{c o}$ can be rewritten as

$$
V_{c o}^{b}=V_{w o}^{b}
$$


Roughly speaking, if both the camera and the target object move, then the relative rigid body motion $g_{c o}$ will be derived from the difference between the camera velocity $V_{w c}^{b}$ and the target object velocity $V_{w o}^{b}$. Hence, the model of the relative rigid body motion from $\Sigma_{c}$ to $\Sigma_{o}$ equals the target object velocity $V_{w o}^{b}$.

\subsection{Estimation Error System}

The relative rigid body motion $g_{c o}$ can not be immediately obtained in the visual feedback system, because the target object velocity is unknown and furthermore can not be measured directly. Hence, we consider the estimation problem of the relative rigid body motion $g_{c o}$. The visual feedback control task requires information of the relative rigid body motion $g_{c o}$. Since the measurable information is only the image information $f\left(g_{c o}\right)$ in the visual feedback system, we consider a visual motion observer in order to estimate the relative rigid body motion $g_{c o}$ from the image information $f\left(g_{c o}\right)$.

Firstly, using the basic representation (4), we choose estimates $\bar{g}_{c o}$ and $\bar{V}_{c o}^{b}$ of the relative rigid body motion and velocity, respectively as

$$
\bar{V}_{c o}^{b}=u_{e}
$$

The new input $u_{e}$ is to be determined in order to drive the estimated values $\bar{g}_{c o}$ and $\bar{V}_{c o}^{b}$ to their actual values.

In order to establish the estimation error system, we define the estimation error between the estimated value $\bar{g}_{c o}$ and the actual relative rigid body motion $g_{c o}$ as

$$
g_{e e}=\bar{g}_{c o}^{-1} g_{c o}
$$

Using the notation $e_{R}\left(e^{\hat{\xi} \theta}\right)$, the vector of the estimation error is defined as $e_{e}:=\left[p_{e e}^{T} e_{R}^{T}\left(e^{\hat{\xi} \theta_{e e}}\right)\right]^{T}$. Note that $e_{e}=0$ iff $p_{e e}=0$ and $e_{R}\left(e^{\hat{\xi} \theta_{e e}}\right)=I_{3}$. Therefore, if the vector of the estimation error is equal to zero, then the estimated relative rigid body motion $\bar{g}_{c o}$ equals the actual relative rigid body motion $g_{c o}$.

Suppose the attitude estimation error $\theta_{e e}$ is small enough that we can let $e^{\hat{\xi} \theta_{e e}} \approx I+\operatorname{sk}\left(e^{\hat{\xi} \theta_{e e}}\right)$. Therefore, using a first-order Taylor expansion approximation, the estimation error vector $e_{e}$ can be obtained from image information $f\left(g_{c o}\right)$ and the estimated value of the relative rigid body motion $\bar{g}_{c o}$ as follows (Fujita et al., 2007) :

$$
e_{e}=J^{+}\left(\bar{g}_{c o}\right)(f-\bar{f})
$$


where $\bar{f}$ is the estimated value of image information. In the same way as the basic representation (4), the estimation error system can be represented by

$$
V_{e e}^{b}=-\operatorname{Ad}_{\left(g_{e e}^{-1}\right)} u_{e}+V_{w o}^{b}
$$

It should be noted that if the vector of the estimation error is equal to zero, then the estimated relative rigid body motion $\bar{g}_{c o}$ equals the actual one $g_{c o}$.

\subsection{Control Error System}

In this subsection, let us consider the dual of the estimation error system, which we call the control error system, in order to establish the visual feedback system. We assume that $g_{w c}$ and $g_{w h}$ can be obtained accurately by a prior calibration procedure, then the estimated value of $g_{h o}$ is calculated as $\bar{g}_{h o}=g_{c h}^{-1} \bar{g}_{c o}$ where $\bar{g}_{c o}$ is the estimated value which discussed in the previous subsection. Here, we define the control error between the actual relative rigid body motion $g_{h o}$ and desired one $g_{d}$ as

$$
g_{e c}=g_{d}^{-1} g_{h o} .
$$

It should be noted that $g_{h o}$ can not be measured directory. Similar to the definition of $e_{e}$, the vector of the control error is defined as $e_{c}:=\left[p_{e c}^{T} e_{R}^{T}\left(e^{\hat{\xi} \theta_{e c}}\right)\right]^{T}$.

Here we have to consider the way of deriving $g_{e c}(9)$, because $g_{h o}$ can not be measured directory. Using $g_{e e}$, the control error can be transformed as

$$
g_{e c}=g_{d}^{-1} g_{h o}=g_{d}^{-1} \bar{g}_{h o}^{-1} \bar{g}_{h o} g_{h o}=g_{d}^{-1} \bar{g}_{h o}^{-1} g_{e e} .
$$

In Equation (10), $g_{d}$ and $\bar{g}_{h o}$ are available information. While the estimation error vector $e_{e}$ can be obtained as Equation

(7), the estimation error matrix $g_{e e}$ cannot be directly obtained, because $g_{e e}$ is defined using non-measurable value $g_{c o}$ as Equation (6). Therefore, we consider the way of deriving $g_{e e}$ from $e_{e}$.

Because of the definition of the estimation error vector $e_{e}$, i.e., $e_{e}=\left[p_{e e}^{T} e_{R}^{T}\left(e^{\hat{\xi} \theta_{e e}}\right)\right]^{T}$, the position estimation error $p_{e e}$ can be derived directly from $e_{e}$. Under the condition $-\frac{\pi}{2} \leq \theta_{e e} \leq \frac{\pi}{2}, \xi \theta_{e e}$ can be derived as follows (Murao et al., 2008):

$$
\xi \theta_{e e}=\frac{\sin ^{-1}\left\|e_{R}\left(e^{\hat{\xi} \theta_{e e}}\right)\right\|}{\left\|e_{R}\left(e^{\hat{\xi} \theta_{e e}}\right)\right\|} e_{R}\left(e^{\hat{\xi} \theta_{e e}}\right)
$$

Hence, $g_{e e}$ can be derived from $e_{e}$ through $\xi \theta_{e e}$. 
The reference of the relative rigid body motion $g_{d}$ is constant in this chapter, i.e., $\dot{g}_{d}=0$, hence, $V_{e c}^{b}=V_{h o}^{b}$. Thus, the control error system can be represented as

$$
V_{e c}^{b}=-\mathrm{Ad}_{\left(g_{e c}^{-1}\right)} \mathrm{Ad}_{\left(g_{d}^{-1}\right)} V_{w h}^{b}+V_{w o}^{b}
$$

This is dual to the estimation error system.

\subsection{Passivity of Visual Feedback System}

Combining (8) and (12), we construct the visual feedback system as follows:

$$
\left[\begin{array}{c}
V_{e c}^{b} \\
V_{e e}^{b}
\end{array}\right]=\left[\begin{array}{cc}
-\mathrm{Ad}_{\left(g_{e c}^{-1}\right)} & 0 \\
0 & -\mathrm{Ad}_{\left(g_{e e}^{-1}\right)}
\end{array}\right] u_{c e}+\left[\begin{array}{l}
I \\
I
\end{array}\right] V_{w o}^{b}
$$

where $u_{c e}:=\left[\left(\operatorname{Ad}_{\left(g_{d}^{-1}\right)} V_{w h}^{b}\right)^{T} u_{e}^{T}\right]^{T}$ denotes the control input. For the design of the visual feedback system, it is assumed that the hand velocity $V_{w h}^{b}$ can be directly chosen. Let us define the error vector of the visual feedback system as $e:=\left[\begin{array}{ll}e_{\mathcal{C}}^{T} & e_{e}^{T}\end{array}\right]^{T}$ which consists of the control error vector $e_{c}$ and the estimation error vector $e_{e}$.

Next, we show an important relation between the input and the output of the visual feedback system.

Lemma 1 (Fujita et al., 2007) : If $V_{w o}^{b}=0$, then the visual feedback system (13) satisfies

$$
\int_{0}^{T} u_{c e}^{T} v_{c e} d t \geq-\beta_{c e}, \forall T>0
$$

where $v_{c e}$ is defined as $v_{c e}:=-e$ and $\beta_{c e}$ is a positive scalar.

Using the following positive definite function, we can prove Lemma 1.

$$
V_{c e}=E\left(g_{e c}\right)+E\left(g_{e e}\right)
$$

where $E\left(g_{a b}\right):=\frac{1}{2}\left\|p_{a b}\right\|^{2}+\phi\left(e^{\hat{\xi} \theta_{a b}}\right)$ and $\phi\left(e^{\hat{\xi} \theta_{a b}}\right):=\frac{1}{2} \operatorname{tr}\left(I-e^{\hat{\xi} \theta_{a b}}\right)$ is the error function of the rotation matrix (see, e.g., (Bullo \& Lewis, 2004)).

The block diagram of the passivity of the visual feedback system is shown in Fig. 2. Let us take $u_{c e}$ as the input and $v_{c e}$ as its output in Fig. 2. Thus, Lemma 1 implies that the visual feedback system (13) is passive from the input $u_{c e}$ to the output $v_{c e}$ as in the definition in (Schaft, 2000). 


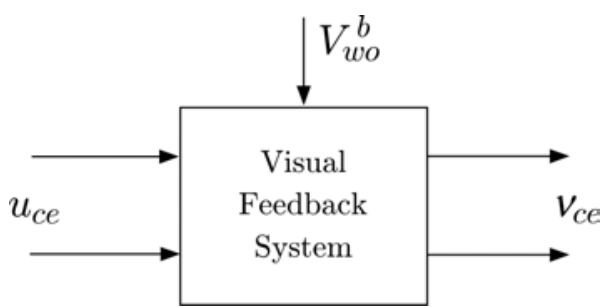

Fig. 2. Block diagram of the visual feedback system

\section{Dynamic Visual Force Feedback Control}

A force is important to complete tasks which need a contact with environments. This section considers the visual force feedback control with a contact force (we call the dynamic visual force feedback control) based on the passivity which is a main contribution in this chapter.

\subsection{Dynamic Visual Force Feedback System}

The dynamics of $n$-link rigid robot manipulators with the end-effector constraint can be written as follows (Liu et al., 1999)

$$
M(q) \ddot{q}+C(q, \dot{q}) \dot{q}+g(q)=\tau+J_{\varphi}(q) \lambda
$$

where $q, \dot{q}$ and $\ddot{q}$ are the joint angle, velocity and acceleration, respectively, $\tau$ is the vector of the input torque. $M(q) \in \mathfrak{R}^{n \times n}$ is the manipulator inertia matrix, $C(q, \dot{q}) \in \mathfrak{R}^{n \times n}$ is the Coriolis matrix and $g(q) \in \mathfrak{R}^{n}$ is the gravity vector. $\lambda \in \Re$ is the contact force, $J_{\varphi}(q) \in \mathfrak{R}^{n}$ is the normalized Jacobian of the kinematic constraint $\varphi(q)=0 \in \Re$ and defined as follows.

$$
J_{\varphi}^{T}(q) \dot{q}=0, \quad J_{\varphi}(q)=\left[\frac{\partial \varphi(q)}{\partial q}\right]^{T} \in \mathfrak{R}^{n}
$$

Equation (16) possesses several important properties which will be used in the sequel. The manipulator dynamics (16) is passive from $\tau$ to $\dot{q}$, that is $\int_{0}^{T} \tau^{T} \dot{q} d t \geq-\beta_{m}$ where $\beta_{m}$ is a positive scalar. Moreover, $\dot{M}(q)-2 C(q, \dot{q})$ is skew-symmetric by defining $C(q, \dot{q})$ using the Christoffel symbols.

Now, we propose the control law for the manipulator as

$$
\tau=M(q) \ddot{q}_{r}+C(q, \dot{q}) \dot{q}_{r}+g(q)+J_{b}^{T}(q) \operatorname{Ad}_{\left(g_{d}^{-1}\right)}^{T} e_{e}-J_{\varphi} \lambda_{d}+u_{s}+J_{\varphi} u_{F}
$$

where

$$
\dot{q}_{r}=Q_{\varphi}(q) \dot{q}_{d}+\alpha J_{\varphi} F_{e}
$$


$\alpha$ is positive constant and

$$
F_{e}:=\int_{0}^{t}\left(\lambda-\lambda_{d}\right) d \tau=\int_{0}^{t} e_{\lambda} d \tau \in \mathfrak{R}
$$

where $e_{\lambda}:=\lambda-\lambda_{d}$ is the force error. Then, the following relation holds with respect to about the force error

$$
\dot{F}_{e}=e_{\lambda}
$$

Because we consider the single point contact in this chapter, the projection matrix $Q_{\varphi}(q)$ can be simply defined as

$$
Q_{\varphi}(q)=I-J_{\varphi}(q) J_{\varphi}^{T}(q)
$$

which arises on the tangent space at the contact surface $\varphi(q)=0$ (Liu et al., 1999).

On the other hand, the body velocity of the hand $V_{w h}^{b}$ is given by

$$
V_{w h}^{b}=J_{b}(q) \dot{q}=J_{b}(q) Q_{\varphi} \dot{q}
$$

where $J_{b}(q)$ is the body manipulator Jacobian (Murray et al., 1994). Moreover, we define the desired body velocity of the hand $u_{h}=\left[v_{u h}^{T} \omega_{u h}^{T}\right]^{T}$ which will be obtained from the visual feedback system. Then, $u_{h}$ can be represented as $u_{h}=J_{b}(q) Q_{\varphi} \dot{q}_{d}$ by using the projection matrix $Q_{\varphi}(q)$.

We define the error vector with respect to the joint velocity of the manipulator dynamics as

$$
s:=\dot{q}-\dot{q}_{r} \in \mathfrak{R}^{n}
$$

Here, we know that the following relation holds (Liu et al., 1999)

$$
J_{\varphi}^{T} s=-\alpha F_{e}
$$

Using (13)(16) and (18), the visual force feedback system with manipulator dynamics (we call the dynamic visual force feedback system) can be derived as follows:

$$
\left[\begin{array}{c}
\dot{s} \\
\dot{F}_{e} \\
V_{e c}^{b} \\
V_{e e}^{b}
\end{array}\right]=\left[\begin{array}{c}
-M^{-1}\left(C s-J_{b}^{T} \operatorname{Ad}_{\left(g_{d}^{-1}\right)}^{T} e_{c}-J_{\varphi} e_{\lambda}\right) \\
e_{\lambda} \\
-\mathrm{Ad}_{\left(g_{h o}^{-1}\right)} J_{b} s \\
0
\end{array}\right]+\left[\begin{array}{cccc}
M^{-1} & M^{-1} J_{\varphi} & 0 & 0 \\
0 & 0 & 0 & 0 \\
0 & 0 & -\mathrm{Ad}_{\left(g_{e c}^{-1}\right)} & 0 \\
0 & 0 & 0 & -\mathrm{Ad}_{\left(g_{e e}^{-1}\right)}
\end{array}\right] u+\left[\begin{array}{l}
0 \\
0 \\
I \\
I
\end{array}\right] w
$$

where 


$$
x:=\left[\begin{array}{c}
s \\
F_{e} \\
e_{c} \\
e_{e}
\end{array}\right] \quad u:=\left[\begin{array}{c}
u_{s} \\
u_{F} \\
\operatorname{Ad}_{\left(g_{d}^{-1}\right)} u_{h} \\
u_{e}
\end{array}\right] w:=V_{w o}^{b},
$$

are defined as the state, the input and the disturbance of the dynamic visual force feedback system, respectively. Here, we formulate the manipulator control problem as follows:

Control problem : For the dynamic visual force feedback system with the eye-to-hand configuration described by (26), design a control input $u$ such that

$$
\lim _{t \rightarrow \infty} s=0, \quad \lim _{t \rightarrow \infty} F_{e}=0, \quad \lim _{t \rightarrow \infty} e_{c}=0 \text { and } \quad \lim _{t \rightarrow \infty} e_{e}=0 .
$$

\subsection{Passivity of Dynamic Visual Force Feedback System}

Before constructing the dynamic visual force feedback control law, we derive an important lemma.

Lemma 2: If $w=0$, then the dynamic visual force feedback system (26) satisfies

$$
\int_{0}^{T} u^{T} v d t \geq-\beta, \forall T>0
$$

where $v:=N x, N:=\operatorname{diag}\left\{I_{n},-\alpha,-I_{6},-I_{6}\right\}$ and $\beta$ is a positive scalar.

Proof: Consider the following positive definite function

$$
V=\frac{1}{2} s^{T} M s+\frac{1}{2} \alpha F_{e}^{2}+E\left(g_{e c}\right)+E\left(g_{e e}\right) .
$$

Differentiating (28) with respect to time yields

$$
\begin{aligned}
\dot{V}=s^{T} M \dot{s} & +\frac{1}{2} s^{T} \dot{M} s+\alpha F_{e} \dot{F}_{e} \\
+ & p_{e c}^{T} e^{\hat{\xi} \theta_{e c}} e^{-\hat{\xi} \theta_{e c}} \dot{p}_{e c}+e_{R}^{T}\left(e^{\hat{\xi} \theta_{e c}}\right) e^{\hat{\xi} \theta_{e c}} \omega_{e c}+p_{e e}^{T} e^{\hat{\xi} \theta_{e e}} e^{-\hat{\xi} \theta_{e e}} \dot{p}_{e e}+e_{R}^{T}\left(e^{\hat{\xi} \theta_{e e}}\right) e^{\hat{\xi} \theta_{e e}} \omega_{e e} \\
= & s^{T}\left[\begin{array}{cccc}
M & 0 & 0 & 0 \\
0 & \alpha & 0 & 0 \\
0 & 0 & \mathrm{Ad}_{\left(e^{\hat{\xi} \theta_{e c}}\right)} \\
0 & 0 & 0 & \mathrm{Ad}_{\left(e^{\hat{\xi}} \theta_{e e}\right)}
\end{array}\right]\left[\begin{array}{c}
\dot{s} \\
\dot{F}_{e} \\
V_{e c}^{b} \\
V_{e e}^{b}
\end{array}\right]+\frac{1}{2} s^{T} \dot{M} s .
\end{aligned}
$$

Observing that the skew-symmetry of the matrices $\hat{p}_{e c}$ and $\hat{p}_{e e}$, i.e., $p_{e c}^{T} \hat{p}_{e c} e^{-\hat{\xi} \theta_{d}} \omega_{u d}$ $=-p_{e c}^{T}\left(e^{-\hat{\xi} \theta_{d}} \omega_{u d}\right)^{\wedge} p_{e c}=0, p_{e e}^{T} \hat{p}_{e e} \omega_{u e}=-p_{e e}^{T} \hat{\omega}_{u e} p_{e e}=0$, the above equation along the trajectories of the system (26) can be transformed into 


$$
\begin{aligned}
& \dot{V}=-s^{T} C \dot{s}+s^{T} s_{b}^{T} \operatorname{Ad}_{\left(g_{d}^{-1}\right)}^{T} e_{c}+s^{T} J_{\varphi} e_{\lambda}+\alpha F_{e} e_{\lambda}-e_{c}^{T} \operatorname{Ad}_{\left(g_{h o}^{-1}\right)} J_{b} s+\frac{1}{2} s^{T} \dot{M} s \\
& +s^{T}\left[\begin{array}{cccc}
M & 0 & 0 & 0 \\
0 & \alpha & 0 & 0 \\
0 & 0 & \mathrm{Ad}_{\left(e^{\hat{\xi} \theta_{e c}}\right)} & 0 \\
0 & 0 & 0 & \mathrm{Ad}_{\left(e^{\hat{\xi} \theta_{e e}}\right)}
\end{array}\right]\left[\begin{array}{cccc}
M^{-1} & M^{-1} J_{\varphi} & 0 & 0 \\
0 & 0 & 0 & 0 \\
0 & 0 & -\mathrm{Ad}_{\left(g_{e c}^{-1}\right)} & 0 \\
0 & 0 & 0 & -\mathrm{Ad}_{\left(g_{e e}^{-1}\right)}
\end{array}\right]+\frac{1}{2} s^{T} \dot{M} s . \\
& =\frac{1}{2} s^{T}(\dot{M}-2 C) s+e_{\lambda}^{T}\left(J_{\varphi}^{T} s+\alpha F_{e}\right)+x^{T} N^{T} u=x^{T} N^{T} u \text {. }
\end{aligned}
$$

Integrating (30) from 0 to $T$, we obtain

$$
\int_{0}^{T} u^{T} v d t V=V(T)-V(0) \geq-V(0)=-\beta
$$

where $\beta$ is a positive scalar that only depends on the initial states of $s, F_{e}, e_{c}$ and $e_{e}$.

The block diagram of the passivity of the $3 \mathrm{D}$ dynamic visual force feedback system is shown in Fig. 3.

Remark 1: The visual feedback system (13) satisfies the passivity property as described in (14). It is well known that the manipulator dynamics (16) also has the passivity. In Lemma 2, the inequality (27) says that the dynamic visual force feedback system (26) is passive from the input $u=\left[u_{s}^{T} u_{F}^{T}\left(A d_{\left(g_{d}^{-1}\right)} u_{h}\right)^{T} u_{e}^{T}\right]^{T}$ to the output $v=\left[s^{T}-\alpha F_{e}^{T}-e_{c}^{T}-e_{e}^{T}\right]^{T}$ as shown in Fig. 3 .

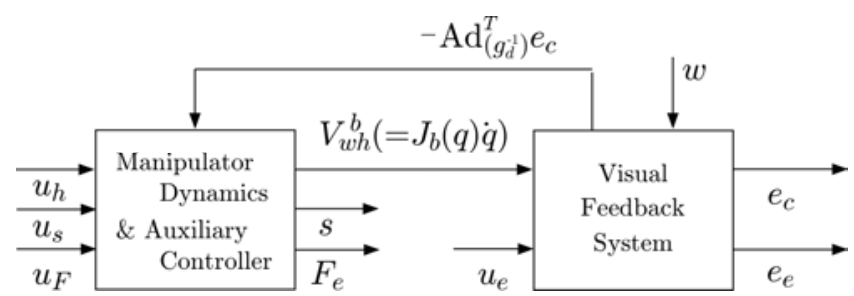

Fig. 3. Block diagram of the 3D dynamic visual force feedback system

\subsection{Passivity-based Dynamic Visual Force Feedback Control}

We now propose the following control input for the interconnected system:

$$
\begin{aligned}
u & =-K v=-K N x \\
K & :=\operatorname{diag}\left\{K_{s}, k_{F}, K_{c}, K_{e}\right\} \in \Re^{n+13}
\end{aligned}
$$

where $K_{s}:=\operatorname{diag}\left\{k_{s 1}, \cdots, k_{s n}\right\}, k_{F}, K_{c}:=\operatorname{diag}\left\{k_{c 1}, \cdots, k_{c 6}\right\}$ and $K_{e}:=\operatorname{diag}\left\{k_{e 1}, \cdots, k_{e 6}\right\}$ denote the positive gain matrices.

Theorem 1: If $w=0$, then the equilibrium point $x=0$ for the closed-loop system (26) and (32)is asymptotic stable. 
Proof: In the proof of Lemma 2, we have already derived that the time derivative of $V$ along the trajectory of the system (26) is formulated as (30). Using the control input (32), (30) can be transformed into

$$
\dot{V}=-x^{T} N^{T} K N x .
$$

This completes the proof.

Theorem 1 shows the stability via Lyapunov method for the dynamic visual force feedback system. It is interesting to note that stability analysis is based on the passivity as described in (27). Our proposed method is valid for the 3D dynamic visual force feedback system, while previous works (Dean-Leon et al., 2006) (Kawai et al., 2007) consider the 2D dynamic visual force feedback control. Hence, we can control not only the position but also the orientation of the robot hand with a contact force in the visual force feedback system.

\section{Simulation Results}

The simulation results on 3DOF planar manipulator as depicted in Fig. 4 are shown in order to understand our proposed method simply, though it is valid for 3D dynamic visual force feedback systems.

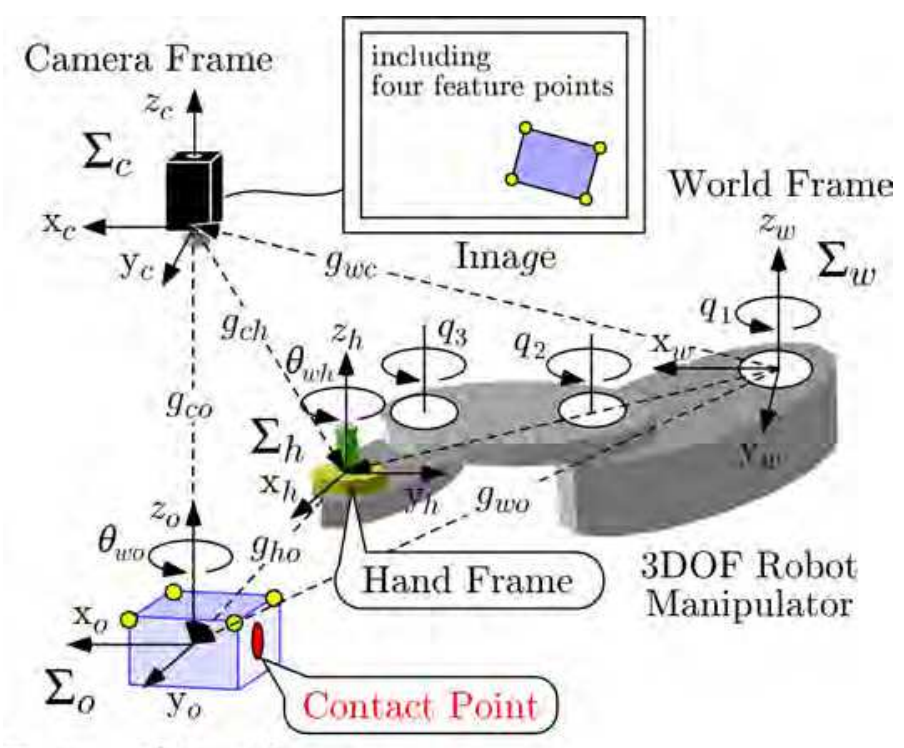

Target Object Frame

Fig. 4. Coordinate frames for dynamic visual force feedback system with three degree of freedom manipulator

We present results for the stability analysis with a static target object. The simulation is carried out with the conditions $p_{w o}=\left[\begin{array}{lll}0.47 & 0.05 & 0\end{array}\right]^{T}[\mathrm{~m}], \quad \xi \theta_{w o}=\left[\begin{array}{lll}0 & 0 & 0\end{array}\right]^{T}[\mathrm{rad}]$, 
$p_{w c}=\left[\begin{array}{lll}0.47 & 0.05 & 1\end{array}\right]^{T}[\mathrm{~m}], \xi \theta_{w c}=\left[\begin{array}{lll}0 & 0 & 0\end{array}\right]^{T}$ [rad $]$. The lengths of the three links of the manipulator are $l_{1}=0.2[\mathrm{~m}], l_{2}=0.2[\mathrm{~m}]$ and $l_{3}=0.1[\mathrm{~m}]$, respectively. The initial angles of the manipulator is set as $q_{1}(0)=-\pi / 2$ [rad], $q_{3}(0)=\pi / 2$ [rad] and $q_{2}(0)=\pi / 2$ [rad]. In other words, the initial relative rigid body motion is $p_{w o}=\left[\begin{array}{llll}0.15 & -0.27 & 0\end{array}\right]^{T}[\mathrm{~m}]$, $\xi \theta_{h o}=\left[\begin{array}{lll}0 & 0 & -\pi / 2\end{array}\right]^{T}$ [rad]. The desired force $\lambda_{d}$ and the desired relative rigid body motion $g_{d}=\left(p_{d}, e^{\hat{\xi} \theta_{d}}\right)$ are given by $\lambda_{d}=5[\mathrm{~N}], p_{d}=\left[\begin{array}{lll}0.03 & 0 & 1\end{array}\right]^{T}[\mathrm{~m}]$ and $\xi \theta_{d}=\left[\begin{array}{lll}0 & 0 & 0\end{array}\right]^{T}$ [rad] in this simulation. The initial errors of force and vision are calculated as $\lambda_{e}=-5[\mathrm{~N}]$, $p_{e c}=\left[\begin{array}{lll}0.12 & -0.27 & 0\end{array}\right]^{T} \quad[\mathrm{~m}], \quad \xi \theta_{e c}=\left[\begin{array}{lll}0 & 0 & -\pi / 2\end{array}\right]^{T} \quad[\mathrm{rad}], \quad p_{e e}=\left[\begin{array}{lll}0.53 & 0.95 & 0\end{array}\right]^{T} \quad[\mathrm{~m}]$ and $\xi \theta_{e e}=\left[\begin{array}{lll}0 & 0 & \pi / 4\end{array}\right]^{T}[\mathrm{rad}]$, respectively. The controller parameters for Equation (32) were empirically selected as $K_{s}=\operatorname{diag}\{10,10,10\}, k_{F}=25, \alpha=1, K_{c}=\operatorname{diag}\{40,40,20,20,20,40\}$ and $K_{e}=50 I_{6}$. The simulation results are shown in Figs. 5-7.

Figs. 5-7 illustrate the control error $e_{c}$, the estimatoin error $e_{e}$, and the contact force $\lambda$, respectively. In Figs. 5 and 6, we focus on the errors of the translations of $x$ and $y$ and the rotation of $z$, because the errors of the translation of $z$ and the rotations of $x$ and $y$ are zeros ideally on the 3DOF planar manipulator. The control error $e_{c}$ and the estimation error $e_{e}$ tended to zero, thus we can confirm that the relative rigid body motion $g_{h o}$ coincided with the desired one $g_{d}$ by using image information. In Fig. 7, the pulse siglnal means the contact transition at around 0.2 [s]. The contact force $\lambda$ tended to 5 [N], i.e., converged to the desired one $\lambda_{d}$. From these figures, the asymptotic stability can be also confirmed.

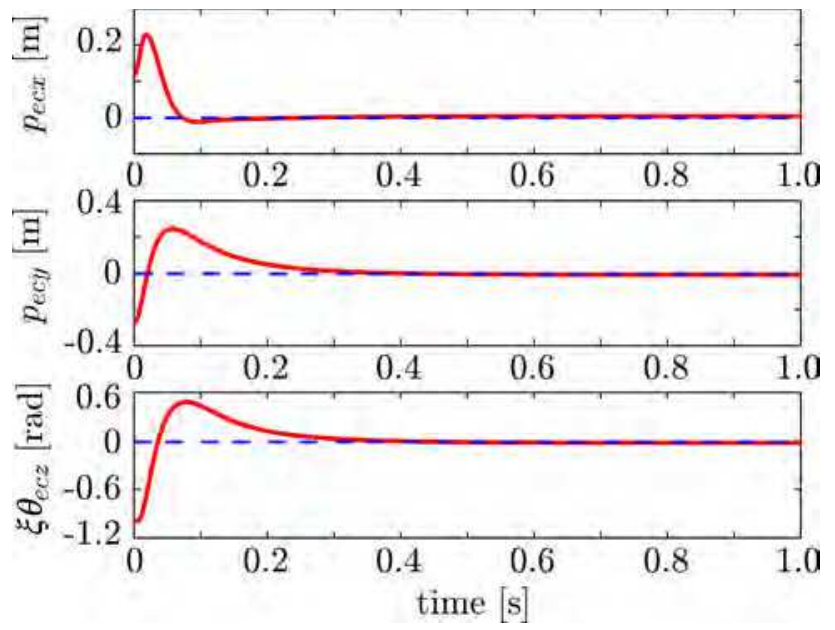

Fig. 5. The control error $e_{c}$ which defined the error between the relative rigid body motion of the robot hand $g_{h o}$ and desired one $g_{d}$. Initial control errors are $p_{e c x}=0.12[\mathrm{~m}]$, $p_{e c y}=-0.27[\mathrm{~m}]$ and $\xi \theta_{e c z}=-\pi / 2[\mathrm{rad}]$, respectively. 


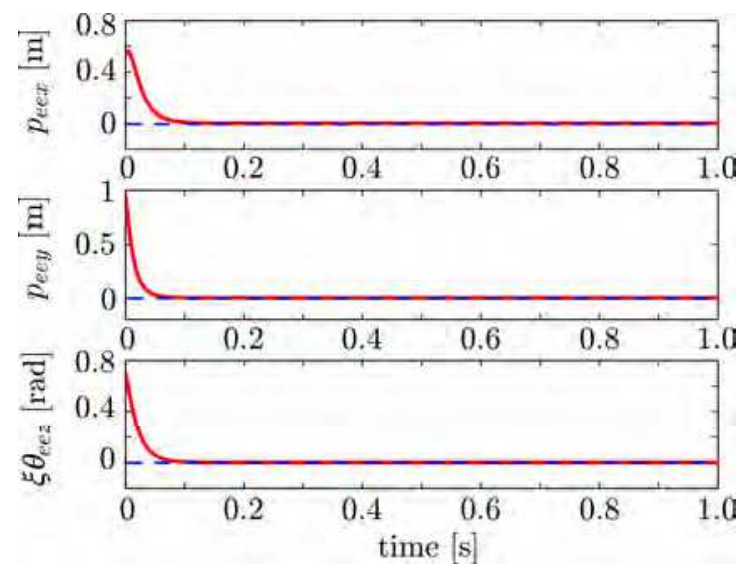

Fig. 6. The estimation error $e_{e}$ which defined the error between the estimated value $\bar{g}_{c o}$ and the actual relative rigid body motion $g_{c o}$. Initial estimation errors are $p_{e e x}=0.53[\mathrm{~m}]$, $p_{\text {eey }}=-0.95[\mathrm{~m}]$ and $\xi \theta_{e e z}=\pi / 4[\mathrm{rad}]$, respectively.

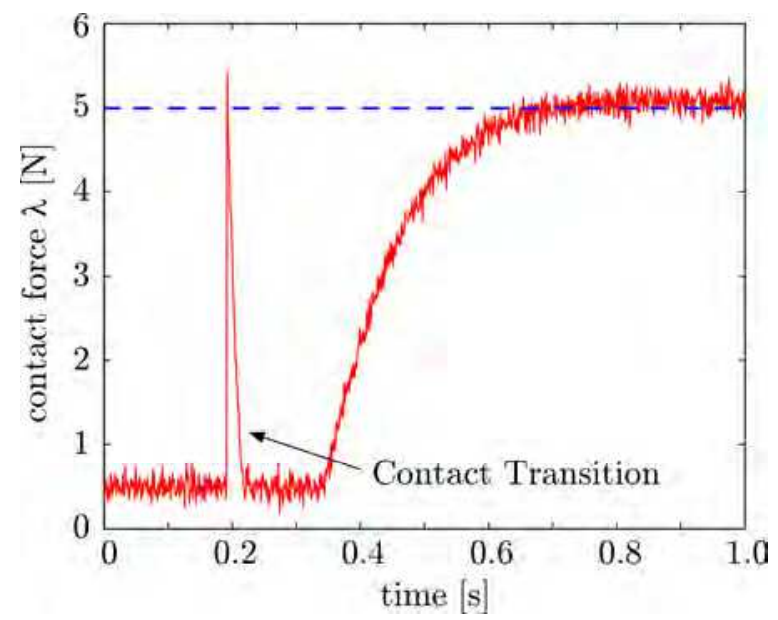

Fig. 7. The contact force trajectory $\lambda$

\section{Conclusions}

This chapter considers 3D visual force feedback control for eye-to-hand systems. In our approach, we can control not only the position but also the orientation of the robot hand with a contact force by using visual information. The proposed method can be regarded as an extension of the hybrid position/force control to the hybrid vision/force control. The main contribution of this chapter is to show that the visual force feedback system has the passivity which allows us to prove stability in the sense of Lyapunov. Both the passivity of the manipulator dynamics and the passivity of the visual feedback system are preserved in 
the visual force feedback system. Finally simulation results are shown to verify the stability of the proposed method.

\section{References}

Baeten, J.; Bruyninckx, H. \& Schutter, J. D. (2003). Integrated Vision/Force Robotic Servoing in the Task Frame Formalism, International Journal of Robotics Research, Vol. 22, No. 10-11, pp. 941-954.

Bullo, F. \& Lewis, A.D. (2004). Geometric Control of Mechanical Systems, Springer-Verlag.

Chaumette, F. \& Hutchinson, S. (2008). Visual Servoing and Visual Tracking, In: Springer Handbook of Robotics, B. Siciliano and O. Khatib (Eds), pp. 563-583. Springer-Verlag.

Chen, J.; Dawson, D. M.; Dixon, W. E. \& Chitrakaran, V. K. (2007). Navigation Functionbased Visual Servo Control, Automatica, Vol. 43, No. 7, pp. 1165-1177.

Dean-Leon, E. C.; Parra-Vega, V. \& Espinosa-Romero, A. (2006). Visual Servoing for Constrained Planar Robots Subject to Complex Friction, IEEE/ASME Trans. on Mechatronics, Vol. 11, No. 4, pp. 389-400.

Fujita, M.; Kawai, H. \& Spong, M. (2007). Passivity-based Dynamic Visual Feedback Control for Three Dimensional Target Tracking: Stability and $L_{2}$-gain Performance Analysis, IEEE Transactions on Control Systems Technology, Vol. 15, No. 1, pp. 40-52.

Kawai, H.; Murao, T. \& Fujita, M. (2007). Passivity-based Visual Force Feedback Control for Planar Manipulators with Eye-in-Hand Configuration, Proc. of the 2007 IEEE Multiconference on Systems and Control, pp. 1480-1485.

Kelly, R.; Carelli, R.; Nasisi, O.; Kuchen, B. \& Reyes, F. (2000). Stable Visual Servoing of Camera-in-Hand Robotic Systems, IEEE Trans. Mechatronics, Vol. 5, No. 1, pp. 39 48.

Liu, Y.; Kitagaki, K.; Ogasawara, T. \& Arimoto, S. (1999). Model-based Adaptive Hybrid Control for Manupilators Under Multiple Geometric Constraints, IEEE Trans. on Control Systems Technology, Vol. 7, No. 1, pp. 97-109.

Murao, T.; Kawai, H. \& Fujita, M. (2008). Predictive Visual Feedback Control with Eyein/to-Hand Configuration via Stabilizing Receding Horizon Approach, Proc. of the 17th IFAC World Congress on Automatic Control, pp. 5341-5346.

Murray, R.; Li Z. \& Sastry, S. S. (1994). A Mathematical Introduction to Robotic Manipulation, CRC Press,

Omote, K. et al., (1999). Self-Guided Robotic Camera Control for Laparoscopic Surgery Compared with Human Camera Control, The American Journal of Surgery, Vol. 177, No. 4, pp. 321-324.

van der Schaft, A. (2000). $L_{2}$ - Gain and Passivity Techniques in Nonlinear Control (2nd ed.), Springer-Verlag,

Xiao, D.; Ghosh, B. K.; Xi, N. \& Tarn, T. J. (2000). Sensor-based Hybrid Position/Force Control of a Robot Manipulator in an Uncalibrated Environment, IEEE Transactions on Control Systems Technology, Vol. 8, No. 4, pp. 635-645,

Yu, S. \& Nelson, B. J. (2001). Autonomous Injection of Biological Cells Using Visual Servoing, In: Experimental Robotics VII, D. Rus and S. Singh (Eds), pp. 169-178, Springer-Verlag. 


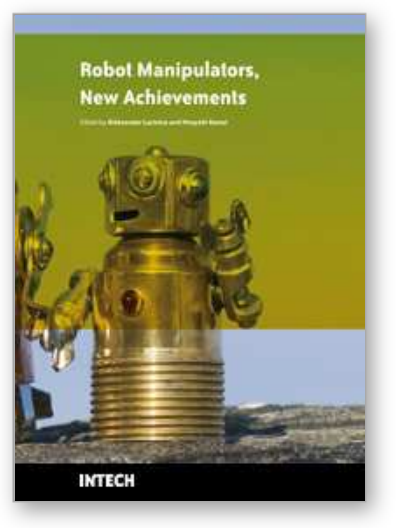

\author{
Robot Manipulators New Achievements \\ Edited by Aleksandar Lazinica and Hiroyuki Kawai
}

ISBN 978-953-307-090-2

Hard cover, 718 pages

Publisher InTech

Published online 01, April, 2010

Published in print edition April, 2010

Robot manipulators are developing more in the direction of industrial robots than of human workers. Recently, the applications of robot manipulators are spreading their focus, for example Da Vinci as a medical robot, ASIMO as a humanoid robot and so on. There are many research topics within the field of robot manipulators, e.g. motion planning, cooperation with a human, and fusion with external sensors like vision, haptic and force, etc. Moreover, these include both technical problems in the industry and theoretical problems in the academic fields. This book is a collection of papers presenting the latest research issues from around the world.

\title{
How to reference
}

In order to correctly reference this scholarly work, feel free to copy and paste the following:

Hiroyuki Kawai, Toshiyuki Murao and Masayuki Fujita (2010). Passivity-based Visual Force Feedback Control for Eye-to-Hand Systems, Robot Manipulators New Achievements, Aleksandar Lazinica and Hiroyuki Kawai (Ed.), ISBN: 978-953-307-090-2, InTech, Available from: http://www.intechopen.com/books/robotmanipulators-new-achievements/passivity-based-visual-force-feedback-control-for-eye-to-hand-systems

\section{INTECH}

open science | open minds

\section{InTech Europe}

University Campus STeP Ri

Slavka Krautzeka 83/A

51000 Rijeka, Croatia

Phone: +385 (51) 770447

Fax: +385 (51) 686166

www.intechopen.com

\section{InTech China}

Unit 405, Office Block, Hotel Equatorial Shanghai

No.65, Yan An Road (West), Shanghai, 200040, China

中国上海市延安西路65号上海国际贵都大饭店办公楼 405 单元

Phone: +86-21-62489820

Fax: +86-21-62489821 
(C) 2010 The Author(s). Licensee IntechOpen. This chapter is distributed under the terms of the Creative Commons Attribution-NonCommercialShareAlike-3.0 License, which permits use, distribution and reproduction for non-commercial purposes, provided the original is properly cited and derivative works building on this content are distributed under the same license. 